\title{
Novel Hydrogels of PVP-CMC and Their Swelling Effect on Viscoelastic Properties
}

\author{
Niladri Roy, Nabanita Saha, Takeshi Kitano, Petr Šaha
}

\begin{abstract}
Polyvinylpyrrolidone (PVP) and carboxy- methyl cellulose (CMC) mixed hydrogels were prepared by heat treatment. The physical characteristics of the hydrogels were studied by Fourier transform infrared spectroscopy and scanning electron microscopy. The swelling study of the hydrogels in water shows remarkable water absorption property. The swelling effect on the rheological behavior of PVP-, PVPCMC-, and CMC-based hydrogels was investigated to judge its application on uneven surface of body. The rheological properties (storage modulus, loss modulus, and complex viscosity) of samples before drying and swelled $(15,30$, and $60 \mathrm{~min})$
\end{abstract}

\section{INTRODUCTION}

Hydrogels or water-containing gels are crosslinked polymeric materials, which maintain a distinct threedimensional structure $^{1}$ and characterized by both hydrophilicity and insolubility in water. ${ }^{2}$ They can be classified on the basis of their sources: natural or synthetic gels; on the nature of the crosslinking: covalent or physical gels; on the nature of the network: homopolymer networks, copolymer networks, interpenetrating networks, or double networks; on the presence of pores: homogeneous (optically transparent) hydrogels, microporous and macroporous hydrogels; on the basis of mechanical spectra: strong and weak hydrogels; on the mechanism of gel formation: pseudo/unstable hydrogels, true/permanent hydrogels; and on their fate in an organism: degrad- able and nondegradable hydrogels. ${ }^{1}$ It was reported in 1960 that hydrogels were the first biomaterials rationally designed for human use. ${ }^{1}$ However, now, these hydrogel biomaterials have a wide range of biomedical applications: drug delivery, contact lenses, control implants, substitutes for skin, ten- were measured against angular frequency and composition. The hydrogel containing PVP/CMC ratio of $20: 80$ appeared to be the best hydrogel from rheological and water absorbent points of view. These properties and low cost of the materials utilized in this work suggest that this hydrogel is a viable alternative product for dressing materials.

Key words: biomaterials; hydrogels; swelling; viscoelastic properties; wound dressing

dons, ligaments, cartilage, and bone. ${ }^{3}$ Hydrogels can be successfully used as a dressing material in medical treatment for burns and wounds. Hydrogels for wound burn dressing were first invented by Rosiak et al. ${ }^{4}$ These materials have many interesting properties such as immediate pain control effect, easy replacement, transparency, barrier against bacteria, good adhesion, easy handling, oxygen permeability, control of drug dosage, absorption, and prevention of loss of body fluids. ${ }^{5}$ Therefore, hydrogel wound dressings could be considered as comparatively more efficient materials than conventional wound dressing materials. From healthcare points of view, hydrogel dressings have become a very interesting field of research for numerous medical applications, thus researchers are giving attention on design and process development of novel hydrogels.

Polyvinylpyrrolidone (PVP)-based hydrogels are well known for their use in wound dressing purposes. Different kinds of PVP wound dressings have already been prepared by radiation crosslinking. ${ }^{5-7}$ PVP is a synthetic polymer with good biocompatibility and can be used as main component of temporary skin covers and wound dressing.718 However, it is also reported that because of inferior mechanical properties and low swelling capacity, the application of PVP hydrogel is not widespread. PVP hydrogels when blended with polysaccharides, their properties improved. ${ }^{910}$ Hydrogels of polysaccharides, on the other hand, have different advantages, such as nontoxicity, biocompatibility, biodegradability, and 
abundance in availability. This is the reason why polysaccharides are obvious choices for investigation as potential wound management aids. Alginic acid/ alginate, cellulose/carboxymethyl cellulose (CMC), chi tin and chitosan, dextrin, heparin, and hyaluronic acid/hyaluronate have been used in wound management aids as dressing materials. ${ }^{11}$ Researchers are now more concentrating on the blends of natural and synthetic polymers for the development of new biomaterials. These modified new polymeric materials, which are prepared by combining natural and synthetic polymers, ${ }^{12}$ have better mechanical properties for biomedical applications than those comprised of single components only. Till today, many researches have been done regarding preparation of hydrogels in combination with synthetic and natural polymers, for example, $\mathrm{PVP} / \mathrm{CMC}{ }^{9}$ dextran/poly (acrylic acid), ${ }^{12}$ starch/PVA, ${ }^{\text {lc }>}$ chitosan/PVP, ${ }^{14}$ chito- san/PVA, ${ }^{15}$ chitosan/poly(ethyleneoxide), ${ }^{16}$ algi- nate/poly(N-isopropyl acrylamide), ${ }^{17}$ and PVP/ PEG/agar. ${ }^{5}$ Among all the natural biomaterials, most abundant polysaccharide is cellulose, a linear chain-shaped polymer joined by (3-1,4-D-glucosidic linkages without any branches. CMC is ether derivative of cellulose, in which original $\mathrm{H}$ atoms of cellulose hydroxyl groups are replaced by carboxymethyl substituent, $\mathrm{CH}_{2} \mathrm{COOH}$. It is highly soluble in water and shows biocompatibility. ${ }^{9}$

All the aforementioned studies about PVP-based hydrogels report that during the formation of hydrogels, radiation-induced crosslinking method is being used. Irradiation technique is very well known for hydrogel preparation and used widely. But, the drawback of this method is that it is expensive and needs extra laboratory setup. However, no study has been reported yet where only moist heat ${ }^{18}$ was applied to initiate crosslinking phenomena within PVP-based hydrogels.

In this article, we discuss the method of preparation of PVP-CMC-based hydrogel and its characterization (physical appearance, physicochemical structure, water uptake capacity, and rheological properties). A series of PVP-CMC hydrogels were prepared with different PVP/CMC ratio to achieve the effective content of composition. It is known that the viscoelastic properties of hydrogels correlate strongly with their microstructures and could pro vide useful information for modulating their performance characteristics. ${ }^{19,20}$ The viscoelastic properties of gel depend on several factors, such as the composition and concentration of the dispersed materials and also the lag time between preparation and measurement. ${ }^{21}$ All these fundamental parameters were investigated and reported on the newly developed hydrogels of PVP-CMC.

\section{EXPERIMENTAL}

A special kind of hydrogel was prepared with PVP, CMC, PEG, agar, and glycerin for biomedical application by moist heat treatment and then characterized following standard methods. As for the components present in the resultant hydrogel PVP and CMC function as base polymers, PEG performs as healing agent, agar acts as gelling agent, and glycerin is playing the role of humectant. ${ }^{18}$

\section{Materials}

PVP K 30 (PVP: molecular weight 40,000), polyethylene glycol 3'000 (PEG: average molecular weight 3015-3685), and agar were supplied by Fluka, Switzerland; carboxymethyl cellulose (CMC: preswollen, microgranular) was purchased from Sigma, USA; glycerin was obtained from Lachema, Czech Republic.

\section{Preparation of hydrogel film ${ }^{18}$}

The PVP-CMC hydrogels were prepared by moist heat treatment using aqueous solution of PVP, CMC, PEG, agar, and glycerin. Five different polymeric solutions were prepared by varying the ratios of PVP and CMC in the following protocol: $0: 100,20: 80,50: 50,80: 20$, and $100: 0$ and keeping the amount of other components constant. The compositions of each component in the hydrogels are shown in Table I. The hydrogel solutions $(100 \mathrm{~mL})$ were prepared in 250-mL sealed glass bottles under physical stimulations (15 lbs pressure and $120^{\circ} \mathrm{C}$ temperature for $20 \mathrm{~min}$ ). Then, the liquids $(20 \mathrm{~mL}$ each) were poured into petridishes $(80-\mathrm{mm}$ diameter) following the solution casting method and allowed to cool at room temperature $\left(22-25^{\circ} \mathrm{C}\right)$. Finally, smooth

TABLE I

Composition of PVP-CMC Hydrogel (Dry Weight Except Glycerin)

\begin{tabular}{|c|c|c|c|c|c|}
\hline Sample No. & PVP (u) & C.MC $(\%)$ & $I^{\prime} F G(\mathrm{~d})$ & Agar $(\%)$ & G G Yretrim $(\%)$ \\
\hline 1 & 0 & ] & 1 & 2 & 1 \\
\hline 2 & 0.2 & 0.8 & 1 & 2 & 1 \\
\hline 3 & 0.5 & 0.5 & 1 & 2 & 1 \\
\hline 4 & 0.8 & 0.2 & 1 & 2 & 1 \\
\hline 5 & l & 0 & 1 & 2 & 1 \\
\hline
\end{tabular}


TABLE II

Physical Appearance of PVP-CMC Hydrogels

\begin{tabular}{|c|c|c|c|c|c|}
\hline \multirow[b]{2}{*}{ Sample } & \multicolumn{2}{|c|}{ Diameter (iпn) } & \multicolumn{2}{|c|}{ 'Ihickness (rтur) } & \multirow[b]{2}{*}{ Motsture content (\%) } \\
\hline & Fresh & Dry & Fresh & Dry & \\
\hline PVP-CMC hydrogels & 80 & $80]$ & $2.9-2.5$ & $0.12-0.15$ & 901795 \\
\hline
\end{tabular}

and whitish round (diameter: $80 \mathrm{~mm}$, thickness: 2- $2.5 \mathrm{~mm}$ ) hydrogels were obtained and designated as "fresh/before dry hydrogels/' whereas thin, smooth, flexible, and transparent films (diameter: $80 \mathrm{~mm}$, thickness: 0.12-0.15 $\mathrm{mm}$ ) were achieved after drying at room temperature and designated as "dry hydrogels." The physical appearance of PVP-CMC hydrogels is shown in Table II.

\section{Measurement of moisture content ${ }^{22}$}

Moisture content of the hydrogels was measured by using eq. (1) in which: $M$, is the moisture content (\%) of material $\mathrm{ft}$, is the wet weight of the sample, and is the weight of the sample after drying. The hydrogel sample was incubated in a mechanical convention type hot air oven at $40^{\circ} \mathrm{C}$ till it assumed a constant weight. Three replicates were taken, and the average value of moisture content was determined.

$$
\left(M_{n}\right) \%=\left[\left(W_{\mathrm{r}:}-W_{d}\right) / W_{w}\right] \times 100 .
$$

\section{Scanning electron microscopy}

Scanning electron microscopy (SEM) analysis was carried out on VEGA II LMU (TESCAN) operating in the highvacuum/secondary electron imaging mode at an accelerating voltage of $5-20 \mathrm{kV}$. The samples were sputter coated with a thin layer of palladium/ gold alloy to improve the surface conductivity and tilted $30^{\circ}$ for better observation. The images were taken at magnification of 100 $\mathrm{x}-10 \mathrm{k} \mathrm{x}$.

\section{Fourier transform infrared spectroscopy}

Pure PVP, pure CMC, and PVP-CMC gels were analyzed by Fourier transform infrared spectroscopy (FTIR). The ATRFTIR spectroscopic analysis was conducted by using a NICOLET 320 FTIR Spectrophotometer with "Omnic" software package over the range $4000-600 \mathrm{~cm}^{-1}$ at room temperature. A uniform resolution of $2 \mathrm{~cm}^{\prime \prime} 1$ was maintained in all cases.

\section{Study of swelling behavior}

The degree of swelling can be described as water absorptivity of the hydrogel. The dry films were Dbtained by drying the hydrogels at room temperature $\left(22-25^{\circ} \mathrm{C}\right)$ until they reached constant weight. Bmall pieces $\left(1 \mathrm{~cm}^{2}\right)$ from the dry films were weighed and immersed in distilled water until $330 \mathrm{~min}$ at room temperature. After the specified time intervals, the water on the swollen gels was wiped off with tissue paper, and the weight of specimens was determined.

The degree of swelling corresponds to the water absorptivity of the material, which is defined by eq. (2), ${ }^{6}$ where $\mathrm{W}_{\mathrm{s}}$ and $V s i_{d}$ are weights of swollen gel and dried gel, respectively.

(Watcr absorptivity) $\%=\left[\left(W_{s}-W_{i l}\right] / W_{i}\right] \times 100$.

\section{Measurement of viscoelastic properties}

The viscoelastic behavior of hydrogels was investigated by using a parallel plate rheometer (ARES; Rheometrics Scientific, USA) testing machine with an "RSI Orchestrator" software package. A 25-mm- diameter parallel plate measuring geometry with a gap of about $2-3 \mathrm{~mm}$ was used, employed at small strain amplitude $(1 \%)$ to maintain the measurements within the linear viscoelastic region. Dynamic frequency sweep tests were carried out at $28^{\circ} \mathrm{C}$ temperature to observe the storage $\left(G^{\prime}\right)$ and loss moduli $\left(G^{\prime \prime}\right)$ as a function of a wide range of angular frequencies (๑) 0.1$100 \mathrm{rad} / \mathrm{s})$. Influences of the PVP- CMC composition on complex viscosity $\left(\left.\mathrm{r}\right|^{*}\right)$, which is calculated by eq. (3), are also discussed.

$$
\eta^{*}-\left(\left(G^{\prime} / 0\right)^{2}+\left(G^{\prime \prime} / \omega\right)^{2}\right)^{1 / 2} .
$$

The rheological properties of the hydrogels were measured several times in different conditions. The viscoelastic properties of the hydrogels just immediately after preparation (designated as fresh/before drying) samples were measured and then after drying the hydrogels the properties were again measured in different swelling time $(15,30$, and $60 \mathrm{~min}$ in water). This measurement was continued up to $60 \mathrm{~min}$, because within 15-min swelling in water, the dry hydrogels regain their shapes and properties like fresh hydrogels, which become useful for application.

\section{RESULTS AND DISCUSSION}

SEM micrographs of the hydrogels

Figure 1 shows the SEM micrographs of the dried samples of hydrogels containing (a) $\mathrm{PVP} / \mathrm{CMC}=100: 0$, (b) $\mathrm{PVP} / \mathrm{CMC}=0: 100$, and (c) $\mathrm{PVP} / \mathrm{CMC}$ 

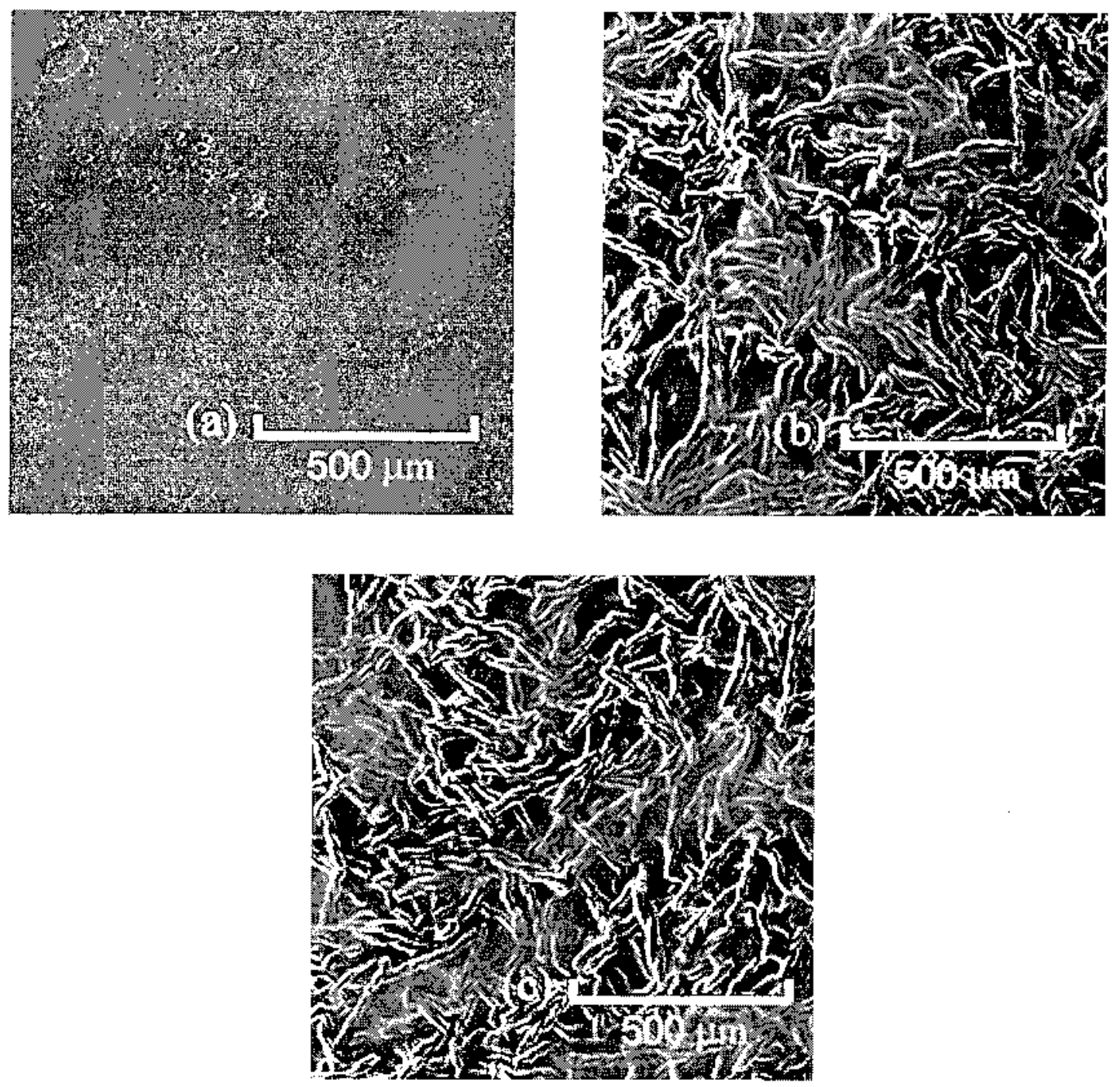

Figure 1 SEM micrographs of (a) PVP/CMC = $100: 0$, (b) PVP/CMC = 0:100, and (c) PVP/CMC = $20: 80$ samples.

$=20: 80$. In Figure 1(a), PVP is dispersed uniformly in the matrix, which cannot be detected under scanning electron microscope. In Figure 1(b), presence of CMC is clearly visible in the form of microgranular structures throughout the hydrogel. Figure 1(c) is the SEM micrograph of the hydrogel containing both PVP $(20 \%)$ and CMC $(80 \%)$. It looks almost similar with Figure 1(b), because PVP and CMC formed an immiscible polymer blend where CMC is distributed uniformly throughout the hydrogel. The images reveal the homogeneity of the components in PVP- CMC blend hydrogel.

FTIR spectroscopy of the hydrogels

FTIR spectra of pure PVP, pure CMC, and PVP- CMC hydrogels are shown in Figure 2. Some charac teristic peaks in PVP spectrum can be ascribed as follows: $1285 \mathrm{~cm}^{-1}$ for $\mathrm{C}-\mathrm{N}$ stretching vibration and $1654 \mathrm{~cm}^{-1}$ for $\mathrm{C}=0$ vibration. ${ }^{9}$ Peak at $1062 \mathrm{~cm}^{-1}$ in the spectrum of $\mathrm{CMC}$ represents 1,4-(3-D-glucoside stretching vibration. ${ }^{23,24}$ In the case of PVP-CMC hydrogels, one single broad peak appears for each hydrogel in the region between 1620 and $1650 \mathrm{~cm}^{\prime \prime} 1$. Formation of this single peak for each hydrogel suggests that there was good interaction among the components (PVP, $\mathrm{CMC}$, agar, PEG, and glycerin) during the formation of PVPCMC hydrogels.

Swelling behavior of the hydrogels

Swelling behavior of the hydrogels was measured in distilled water to evaluate the extent of their water uptake property. Huge water retention capacity is 


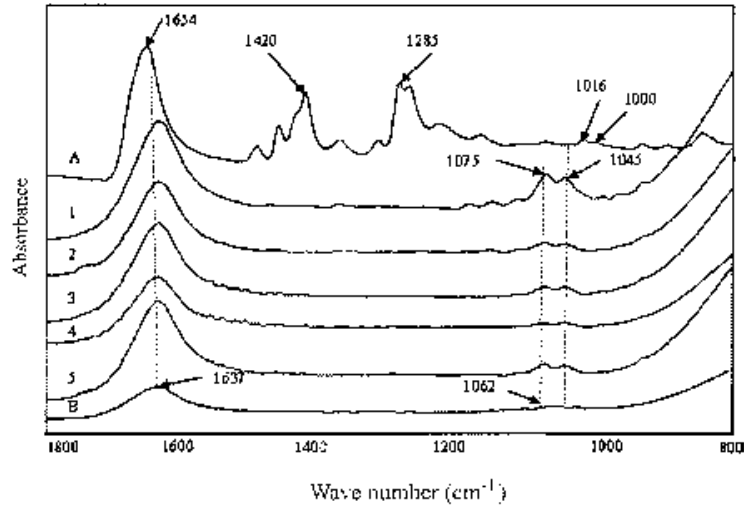

Figure 2 FTIR spectra of (A) pure PVP, (B) pure CMC, and (1-5) PVPCMC hydrogels. (1) $\mathrm{PVP} / \mathrm{CMC}=0: 100,(2) \mathrm{PVP} / \mathrm{CMC}=20: 80$, (3) $\mathrm{PVP} / \mathrm{CMC}=50: 50$, (4) $\mathrm{PVP} / \mathrm{CMC}=80: 20$, and (5) $\mathrm{PVP} / \mathrm{CMC}=100: 0$.

one of the main characteristics of hydrogels. Figure 3 represents the variation in water absorption property of the hydrogels, which depends on the PVP- CMC ratio. PVP of its own accord does not have good absorption capacity, but when blended with $\mathrm{CMC}$, its swelling property increases significantly. ${ }^{9,10}$ As the percentage of $\mathrm{CMC}$ increases, water uptake capacity also increases almost proportionately. The time required to reach equilibrium swelling varies for different hydrogels, but after around $5 \mathrm{~h}$ all hydrogels would have reached equilibrium condition. When there is a network of the chains of polyelectrolyte containing ionizable groups, the mobile counterions present in the gels develop a large swelling pressure because of some intermolecular nonco- valent interaction, such as coulombic repulsion, hydrogen-bonding, and polar forces. ${ }^{9,25}$ Thus, the polymeric interactions increase and cause a very high sorption rate. Because of this reason the swelling capacity of the hydrogels increases noticeably with the variation of the content of CMC. $^{9}$

\section{Rheological behavior of the hydrogels}

There are basically two types of theories exist to explain rheological behavior. Macroscopic stress- strain-time relationships are being used in phenomenological theories; while molecular theories provide evidence of viscoelastic behavior from molecular structure and conformation. ${ }^{26}$

Figure 4(a) shows storage modulus $G$ and loss modulus $G^{\prime \prime}$ versus angular frequency co plots for some typical samples (fresh samples). For all the samples, storage modulus is higher than loss modulus and they all show a similar trend in behavior except $G$ of the sample PVP/CMC $=100: 0$. Angular frequency does not affect so much on storage modulus showing similar to rubbery plateau behav ior. In case of $\log G^{\prime \prime}$ versus $\log$ oo plots, initially the values of loss modulus decrease with respect to angular frequencies, but then the values become steady. Decrease in the value of loss modulus in the beginning may be due to some changes in internal structure of the samples. This seems to be a typical behavior of the gels, which were achieved in this work as well as reported for the different types of gels. ${ }^{27}$

Figure 4(b) shows the effect of angular frequency (oo) on storage modulus $\left(G^{i}\right)$ and loss modulus $\left(G^{\prime \prime}\right)$ for swelled samples of 15 and $60 \mathrm{~min}$ of swelling, where reported about only two kinds of hydrogels having PVP/CMC ratio $50: 50$ and $20: 80$. Swelled samples of 15 and 30 min show almost same trend, thus samples of 15 and $60 \mathrm{~min}$ of swelling have been taken into consideration to avoid complicacy. All the samples show higher elastic property than viscous property except 60-min swelled sample of PVP/CMC $=50: 50$. It seems that the composition of the hydrogels has a great effect on their properties. In each case, the hydrogels of $\mathrm{PVP} / \mathrm{CMC}=20: 80$ have greater values in storage modulus $(G)$ and loss modulus $\left(G^{1}\right)$ than that of the hydrogels of $\mathrm{PVP} / \mathrm{CMC}=50: 50$, irrespective of swelling time. Those values may be obtained because of the PVP/ CMC $=20: 80$ blends well as a result creates more numbers of crosslinking. Swelling time also affects the behavior of the hydrogels. As the swelling time increases both the $G$ and $G^{\prime \prime}$ values increase. That may be due to the formation of some more hydrogen bonds within the hydrogels that provide extra mechanical strength to the material. However, the hydrogel that contains PVP/CMC $=50: 50$ shows some abnormality in $G$ values after $60 \mathrm{~min}$ of swelling. Storage moduli of this hydrogel decrease with swelling. The hydrogel containing $\mathrm{PVP} / \mathrm{CMC}=20: 80$ after

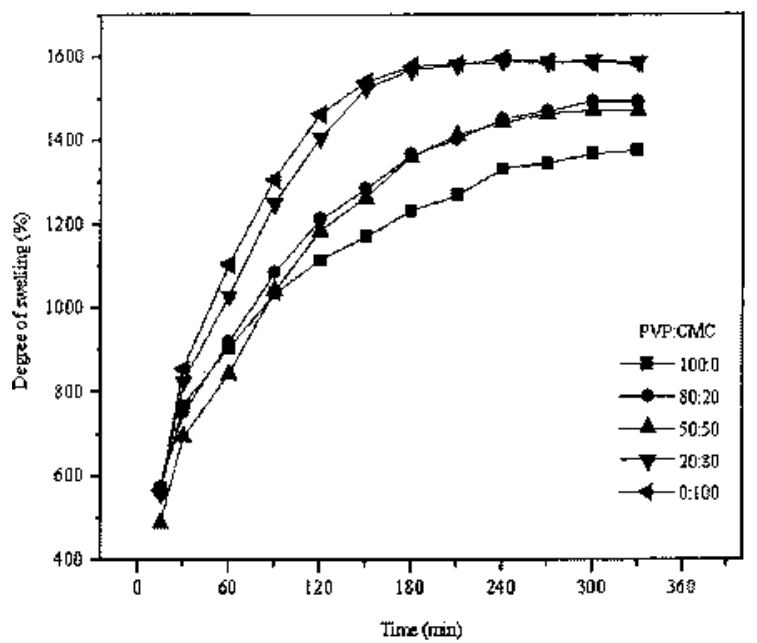

Figure 3 Swelling behavior of hydrogels. 


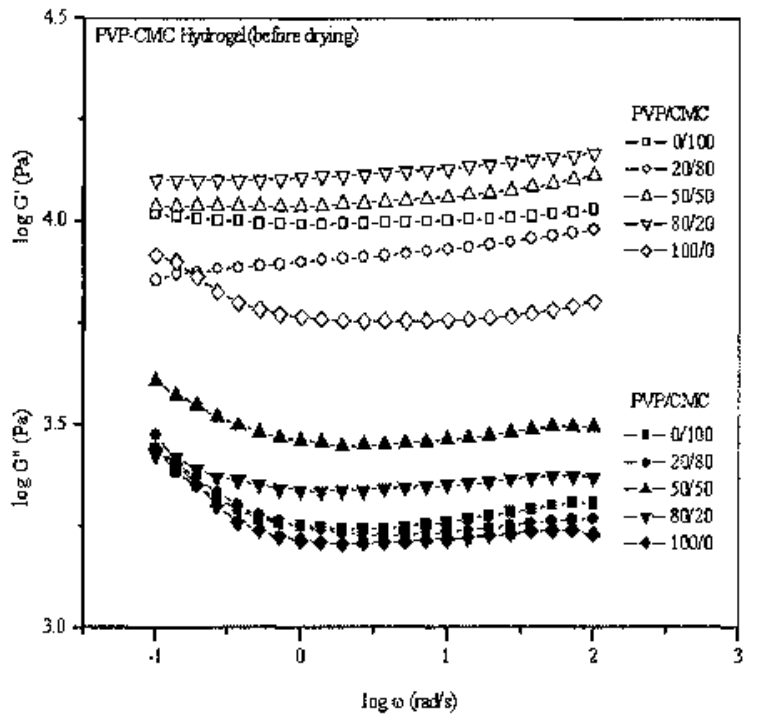

(a)

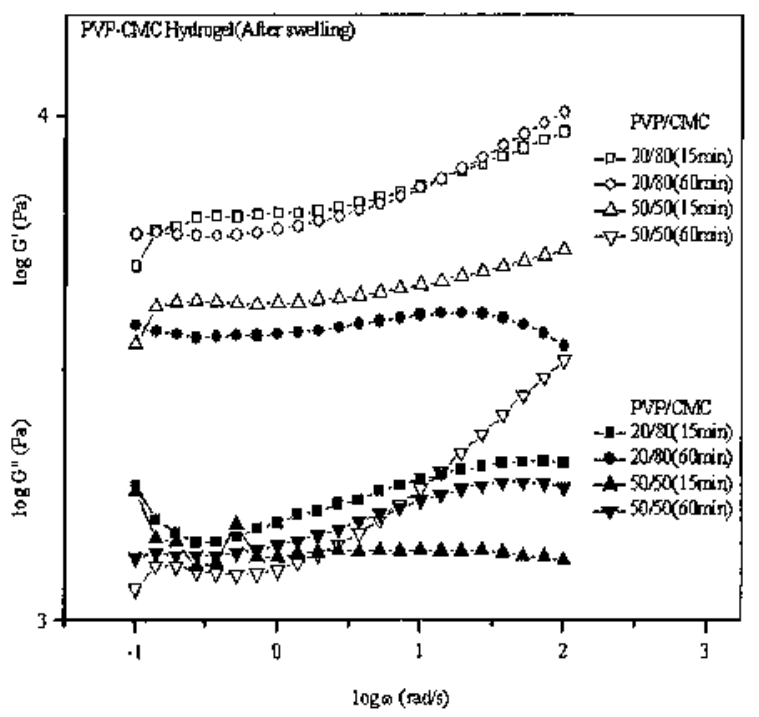

(b)

Figure 4 (a) Effect of angular frequency (co) on storage modulus (G;) and loss modulus (G") for fresh samples (before drying), (b) Effect of angular frequency (co) on storage modulus (G') and loss modulus (G") for swelled samples after 15 and $60 \mathrm{~min}$.

$60 \mathrm{~min}$ of swelling shows best behavior in rheological property with high elasticity.

Figures 5-7 show the viscoelastic behavior of the hydrogels with variation in swelling time $(15,30$, and 60 $\mathrm{min})$. These figures represent the effect of composition and swelling time on storage modulus $\left(G^{\prime}\right)$, loss modulus (G"), and complex viscosity $\left(\left.\mathrm{r}\right|^{*}\right)$ consecutively at different angular frequencies. In all these three cases, the variation in go does not show
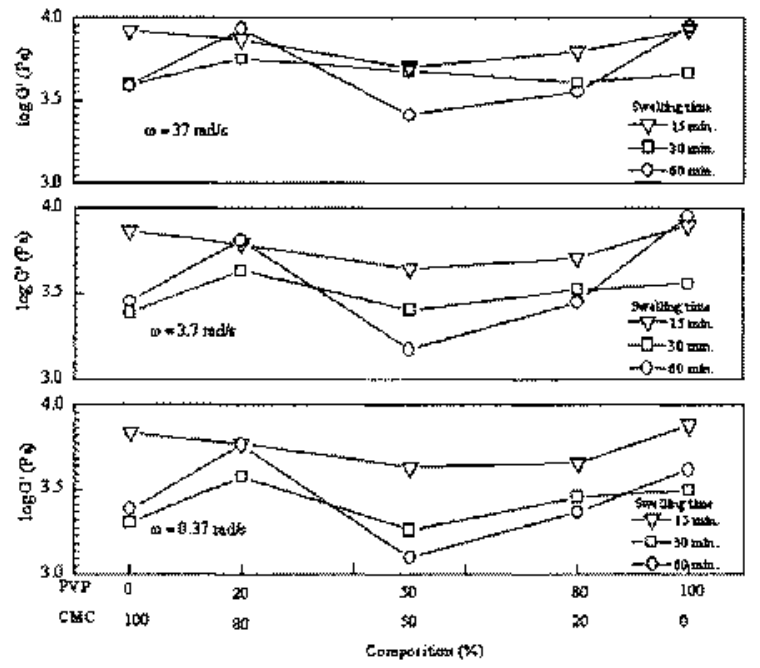

Figure 5 Effect of composition and swelling time on storage modulus $\left(G^{\prime}\right)$ at different angular frequencies (co) for the samples prepared with different swelling time.

much influence on the properties of the hydrogels. That indicates that the hydrogels consist of very good crosslinking and provide mechanical stability.

From Figures 5 and 6, it can be seen in all cases that storage modulus is significantly higher than loss modulus. Angular frequencies do not affect much on behavior, but swelling time shows great effect on it. This result supports that hydrogels are showing high elastic property. This property is very much necessary for hydrogels from the application point of view.
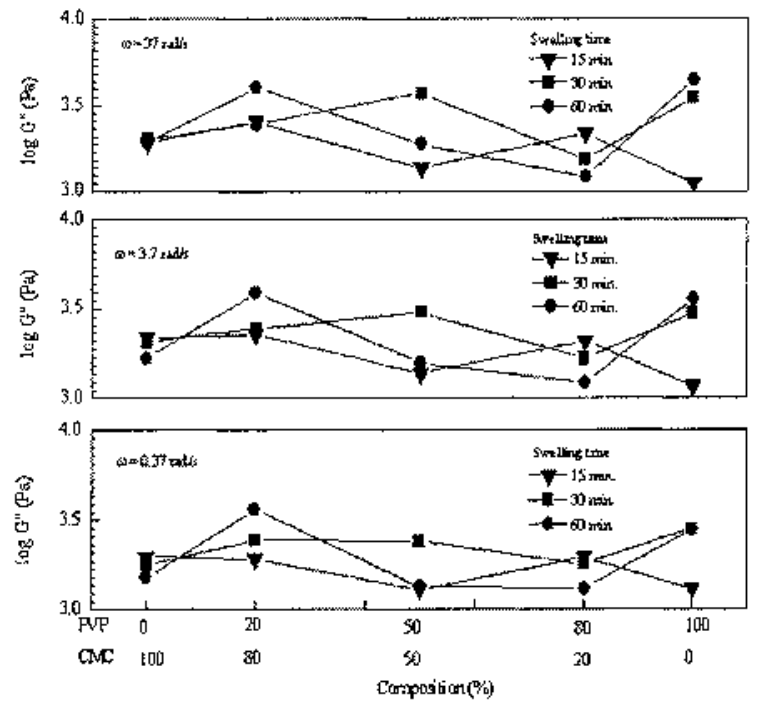

Figure 6 Effect of composition and swelling time on loss modulus (G") at different angular frequencies (go) for the samples prepared with different swelling time. 


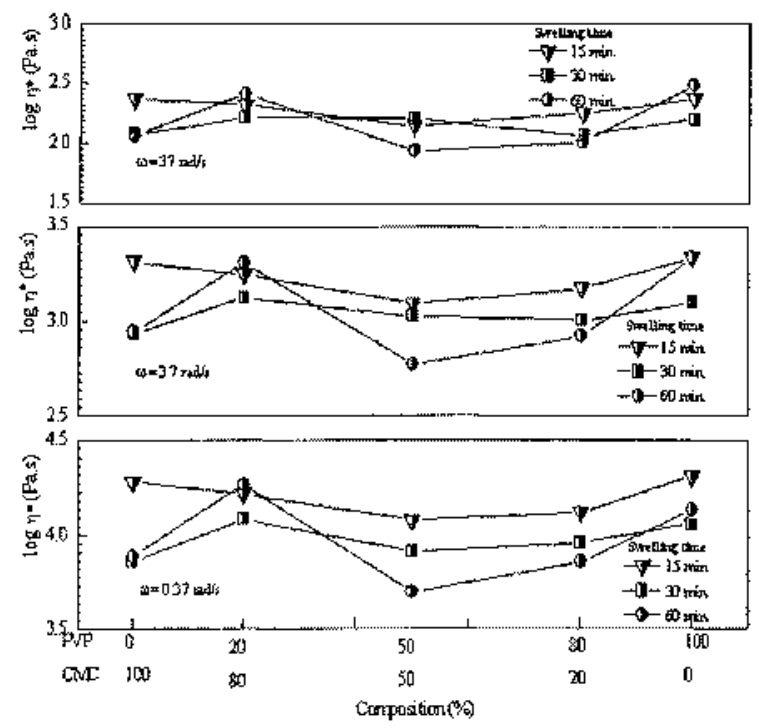

Figure 7 Effect of composition and swelling time on complex $\operatorname{viscosity}\left(\left.r\right|^{*}\right)$ at different angular frequencies (C) for the samples prepared with different swelling time.

In Figure 7 , the complex viscosity $\left(\left.\mathrm{r}\right|^{*}\right)$ graph represents how viscous and elastic properties influence on flow behavior of the hydrogels. As we can see from the previous figures that $G^{\prime}$ is always significantly higher than $G^{\prime \prime}$ for each samples, so it can be suggested that the influences of storage modulus $\left(G^{\prime}\right)$ is predominant on the flow behavior of the hydrogels, and the flow of the gels investigated here is more elastic, rather than viscous.

The hydrogels are generally formed by physical and chemical crosslinking and result in a three- dimensional (3D) network. The viscoelastic behavior of these gels depends strongly on the mechanism of gel formation. Actually, hydrogels are classified into two groups: physical and chemical gels. In physical gels, the polymer chains are connected by electrostatic force, hydrogen bonds, hydrophobic interactions, or chain entanglements, as a result they form pseudo/unstable gels, which can be converted into polymer solutions by heating. Whereas, in chemical gels, covalent bonds linking the chains of polymers thus form true/permanent gels. ${ }^{8,25}$ In this article, we deal with the formation of pseudo polymer network by heat treatment. The formation of the three-dimensional network structure and its mechanical property is influenced here by molecular secondary forces such as hydrogen-bonding, molecular entanglements, and hydrophobic interactions. In this case, agar also acts as a natural gelling agent. Agar can form hydrogel on cooling the solution, because the gel is formed due to the formation of helices and its association. ${ }^{8}$
Generally, typical strong gel spectra demonstrate two nearly horizontal straight lines; $G^{\prime}$ shows 1-2 orders of magnitude higher than $G^{\prime \prime} .{ }^{26}$ Similar rheo- logical behavior was also observed in another types of gels, i.e., carbomer gels for ophthalmic use. ${ }^{2}$ All the hydrogels investigated in this study also show higher elasticity/storage modulus $\left(G^{7}\right)$ than viscosity/loss modulus (G"), and the frequency dependence was insignificant irrespective of hydrogel composition. These data strongly support the formation of PVP$\mathrm{CMC}$ hydrogels and establish them as strong gels. From Figures 5-7, it is clear that the hydrogels having the composition PVP/CMC $=20: 80$ show the higher values of $G^{\prime}, G^{\prime \prime}$, and $\left.r\right|^{*}$ as well as have consistency with swelling time. Higher storage modulus indicates elevated elastic property, but among all the test samples, the value of elasticity modulus is highest for the PVP/CMC $=20: 80$ hydrogels. Therefore, $\mathrm{PVP} / \mathrm{CMC}=20: 80$ can be considered as the best blend ratio from the swelling as well as rheological points of view.

\section{CONCLUSIONS}

Five types of hydrogels of PVP/CMC blends with different compositions (PVP/CMC $=0: 100,20: 80,50: 50,80: 20$, and $100: 0, \mathrm{w} / \mathrm{w}$ ) were prepared from PVP and CMC aqueous solution by means of moist heating. Agar plays a significant role for the formation of the hydrogels as agar is a natural gelling agent. PVP/CMC blend hydrogels have much better swelling behavior than that of pure PVP hydrogel and better dynamic mechanical properties than that of pure CMC hydrogel. Among them, the hydrogel with $\mathrm{PVP} / \mathrm{CMC}=20: 80$ shows the best result in water absorption property as well as in rheological properties with variation in swelling time. In addition to the excellent swelling property and good elasticity, the hydrogels are having some other properties, which suggest that they can be used as wound dressing material. They are flexible, semitransparent, give cool feelings, easy to remove, and permit the observation of the healing process. Moreover, they seem very promising for commercialization because of the cheapness and abundance of the materials and simple low-cost methodology for preparation.

\section{References}

1. Kopecek, J.; Yang, J. Polym Int 2007, 56, 1078.

2. Schacht, E. H. J Phys Conf Ser 2004, 3, 22

3. Stammen, J. A.; Williams, S.; Ku, D. N.; Guldberg, R. E. Biomaterials 2001, 22, 799.

4. Rosiak, J.; Rucinska-Rybus, A.; Pekala, W. U.S. Pat. 4,871,490 (1989).

5. Higa, O. Z.; Rogero, S. O.; Machado, L. D. B.; Mathor, M. B. Lugao, A. B. Radiat Phys Chem 1999, 55, 705. 
6. Park, K. R.; Nho, Y. C. Radiat Phys Chem 2003, 67, 361.

7. http://www.dae.gov.in/publ/betrlife/health/hydrog el.pdf (accessed December 2009).

8. Benamer, S.; Mahlous, M.; Boukrif, A.; Mansouri, B.; Youcef,

S. L. Nucl Instrum Methods Phys Res Sect B 2006, 248, 284.

9. Wang, M.; Xu, L.; Hu, H.; Zhai, M.; Peng, J.; Nho, Y.; Li, J.; Wei,

G. Nucl Instrum Methods Phys Res Sect B 2007, 265, 385.

10. Zhao, L.; Xu, L.; Mitomo, H.; Yoshii, F. Carbohydr Polym 2006, 64, 473 .

11. Sen, M.; Avci, E. N. J Biomed Mater Res 2005, 74, 2005.

12. Cascone, M. G.; Polacco, G.; Lazzeri, L.; Barbani, N. J Appl Polym Sci 1997, 66, 2089.

13. Zhai, M.; Yoshii, F.; Kume, T.; Hashim, K. Carbohydr Polym 2002, 50, 295.

14. Risbud, M. V.; Bhat, S. V. J Mater Sci: Mater Med 2001, 12, 75.

15. Li, M.; Cheng, S.; Yan, H. Green Chem 2007, 9, 894.

16. Khoo, C. G.; Frantzich, S.; Rosinski, A.; Maria, S. M.; Hoogstraate, J. Carbohydr Polym 2003, 55, 47.
17. Ju, H. K.; Kim, S. Y.; Lee, Y. M. Polymer 2001, 42, 6851.

18. Saha, P.; Saha, N.; Roy, N. Czech. Pat. RIV/70883521:28110/ 08:63507001 (2008).

19. Weng, L.; Chen, X.; Chen, W. Biomacromolecules 2007, 8, 1109.

20. Zandraa, O.; Saha, N.; Pavlinek, V.; Kitano, T.; Saha, P. The PPS24 Annual Meeting, Salerno, Italy, 2008.

21. Mourtas, S.; Haikou, M.; Theodor op oulou, M.; Tsakiroglou, C.; Antimisiaris, S. G. J Colloid Interface Sci 2008, 317, 611.

22. http://compost.css.cornell.edu/calc/moisture_content. html (accessed December 2009).

23. Biswal, D. R.; Sing, R. P. Carbohydr Polym 2004, 57, 379.

24. Barbucci, R.; Magnani, A.; Consumi, M. Macromolecules 2000, 33, 7475 .

25. Rosiak, J. M.; Ulanski, P. Radiat Phys Chem 1999, 55, 139.

26. Marrtinez-Ruvalcaba, A.; Chomet, E.; Rodrigue, D. Carbohydr Polym 2007, 67, 586

27. Edsman, K.; Carlfors, J.; Harju, K. Int J Pharm 1996, 137, 233. 